\title{
Appropriateness Evaluation of Energy Saving Techniques for External Envelope of Residential Buildings Based on Value Engineering Theory
}

\author{
Tianqing Shen*, Xingwei Shen \\ School of Urban Construction and Transportation, Hefei University, Hefei 230000, Anhui, China
}

Corresponding Author Email: shtq@hfuu.edu.can

https://doi.org/10.18280/ijht.390610

Received: 20 September 2021

Accepted: 18 November 2021

\section{Keywords:}

appropriateness, value engineering, energysaving insulation

\begin{abstract}
Appropriate technology aims to achieve the optimal comprehensive benefits. The improvement of comprehensive benefits depends on the betterment of every benefit and the overall balance between benefits. Through value engineering evaluation, this paper transforms qualitative method into a quantitative method, and uses the new method to compare the comprehensive benefits of technical schemes for energy saving of external envelope of residential buildings, laying the basis for improving technical schemes. Multiple functional indices were determined, and organized into a hierarchical structure. The relative weight of each index was calculated through analytic hierarchy process (AHP). Drawing on the principle of value engineering, function coefficient, cost coefficient, and value coefficient were calculated for examples, and subjected to empirical comparison and analysis. After that, the technical schemes were contrasted, analyzed, and improved, according to the numerical value of the value coefficient. The proposed evaluation system provides a theoretical guide for making investment decisions on energysaving residential buildings, and avoiding blind investment.
\end{abstract}

\section{INTRODUCTION}

By 2040, the global consumption of primary energy is expected to be $32 \%$ higher than that in 2017 [1]. In China, buildings alone consume more than $20 \%$ of the total energy utilized in the country [2].

Energy-saving buildings have long been a hot topic among various parties [3-5]. The public sector mainly eyes how to realize energy goals, and ensure the energy-efficiency of incentives, risk guarantees, and regulations [6]. Meanwhile, ordinary citizens are concerned with sharing the information about retrofitting benefits, consulting access, initial investment cost, and financial support $[7,8]$. As for the private sector, the most interesting issues are financial profits, risk guarantees, and payback time [9].

In the design circle, the research of building energy-saving mostly stops at the technology level, with the aim to improve the energy efficiency of buildings through technology application. The thermal performance of envelopes is a key impactor of building energy consumption [10-12]. Buildings will consume much fewer energy, if their envelopes have a better thermal performance $[13,14]$. In the external envelope of residential buildings, the energy-saving insulation system relies primarily on the outer wall to save energy [15-17]. The outer wall insulation can effectively reduce the airconditioning load caused by the heat transfer of building walls [18-21]. The type and thickness of insulation material directly bear on the energy-saving effect and economic benefit. Hence, outer wall insulation is an important measure for building energy-saving [22-32].

The relevant studies by governments and enterprises attempt to encourage the development of high and new technology, overlooking the energy and resources consumed by energy-saving materials and equipment. Neither have they paid sufficient attention to economic foundation, production and transport of energy-saving materials, or the environmental impact of such materials. To solve the problem, it is necessary to implement appropriate technology, which aims to achieve the optimal comprehensive benefits, involving technical benefit, economic benefit, and environmental benefit.

With the introduction of the relevant national policies, codes, and standards, energy-saving residential buildings boast broad market prospects. However, there is no systematic research into the evaluation of composite benefits for the energy-saving insulation system of the external envelope of residential buildings. On the one hand, the comprehensive benefits of different combinations of insulation structures are not compared systematically, under the same energy-saving standard. On the other hand, different regions have different economic carrying capacities, facing the current national situation; it remains unclear which energy-saving rate leads to the optimal comprehensive benefits. The previous research of building energy-saving focuses on the energy-saving design of buildings, application of energy-saving products, and implementation of energy-saving techniques. Nevertheless, very few scholars have studied the economy and appropriateness for the application of energy-saving techniques [33].

As an applied management technology, value engineering is often applied to building design to discover, analyze, and solve contradictions [34-37]. When it comes to the energysaving optimization design for the external envelope insulation system of residential buildings, the principle of value engineering helps to disclose the relationship between the functions and cost of the external envelope insulation system, identify the key nodes that affect the system cost 
effectiveness, find the most cost effective combination of insulation techniques, and thereby optimize the design, control the overall cost, and improve the cost effectiveness (value) of the project. Therefore, the relationship between technology and economy should be handled correctly: the reasonable technical requirements must not be overlooked for the sake of cost control, or the project will not meet the functional needs; neither is it acceptable to emphasize technology over economy, which results in too futuristic design, and insufficient use or waste of resources [38-41].

Taking the value engineering theory as an evaluation tool, this paper combines building technology, building economy, and building eco-environment, and makes a comprehensive analysis of the appropriate energy-saving techniques for the external envelope structure of energy-saving residential buildings in hot summer cold winter regions. The appropriateness of energy-saving techniques was evaluated systematically on a broad front. The relevant results enrich the domestic theories on energy-saving technology and economy, and guide the practice of building designers. The main research contents are as follows:

(1) Determine functional indices, and compile them into a hierarchical structure.

(2) Construct a judgement matrix.

(3) Compute the relative weight of each functional index through analytic hierarchy process (AHP).

(4) Drawing on the principle of value engineering, empirically compare and analyze function coefficient, cost coefficient, and value coefficient of examples, and contrast, analyze, and improve the technical schemes according to the numerical value of the value coefficient.

\section{DETERMINATION OF FUNCTIONAL INDICES}

\subsection{Index weighting}

According to the features of functional value evaluation for residential communities, and the strengths/weaknesses and applicable scopes of the relevant methods, this paper chooses the AHP to determine the weight of each functional index, and establishes a hierarchical structure for the appropriate technology evaluation of the external envelope insulation system of residential buildings:

(1) Goal layer

From the previous analysis on the relevant concepts, appropriate technology pursues the optimal comprehensive benefits. To examine the comprehensive benefits of the energy-saving techniques for the envelope structure, it is necessary to establish a hierarchical analysis structure.

(2) Criterion layer

The appropriate technology was examined comprehensively from three aspects: technology, economy, and ecology. Referring to Performance Evaluation Method and Index System for Commercial Residential Buildings and Todd and Simpson's research [42], the key properties of residential buildings were defined as applicability, safety, durability, economy, and ecology. As physical manifestations of the essential attributes, these properties basically cover all the functions of residential buildings. Hence, this paper defines the criteria under the goal of comprehensive benefits of appropriate techniques as applicability, safety, durability, economy, and ecology.

(3) Alternative layer
The applicability criterion consists of the following alternatives: heat preservation and insulation, lighting and ventilation, compressive strength, water absorption, and soundproof performance. The safety criterion consists of the following alternatives: structural safety, flame retardancy, construction safety, toxic and harmful substance emissions, and health hazard. The durability criterion consists of the following alternatives: material service life, material corrosion resistance, waterproof and leakproof, and air permeability. The economy criterion consists of the following alternatives: costeffectiveness, industrialization level, construction convenience, construction period, construction controllability, and technical maturity and innovation. The ecology criterion consists of the following alternatives: physical-chemical energy, renewable material utilization, locality, ecological destruction, and waste recycling.

In this way, the authors established the hierarchy of evaluation indices for the appropriate techniques of the energy-saving insulation system of the external envelope of residential buildings (Figure 1), and assigned a code for each evaluation index (Table 1).

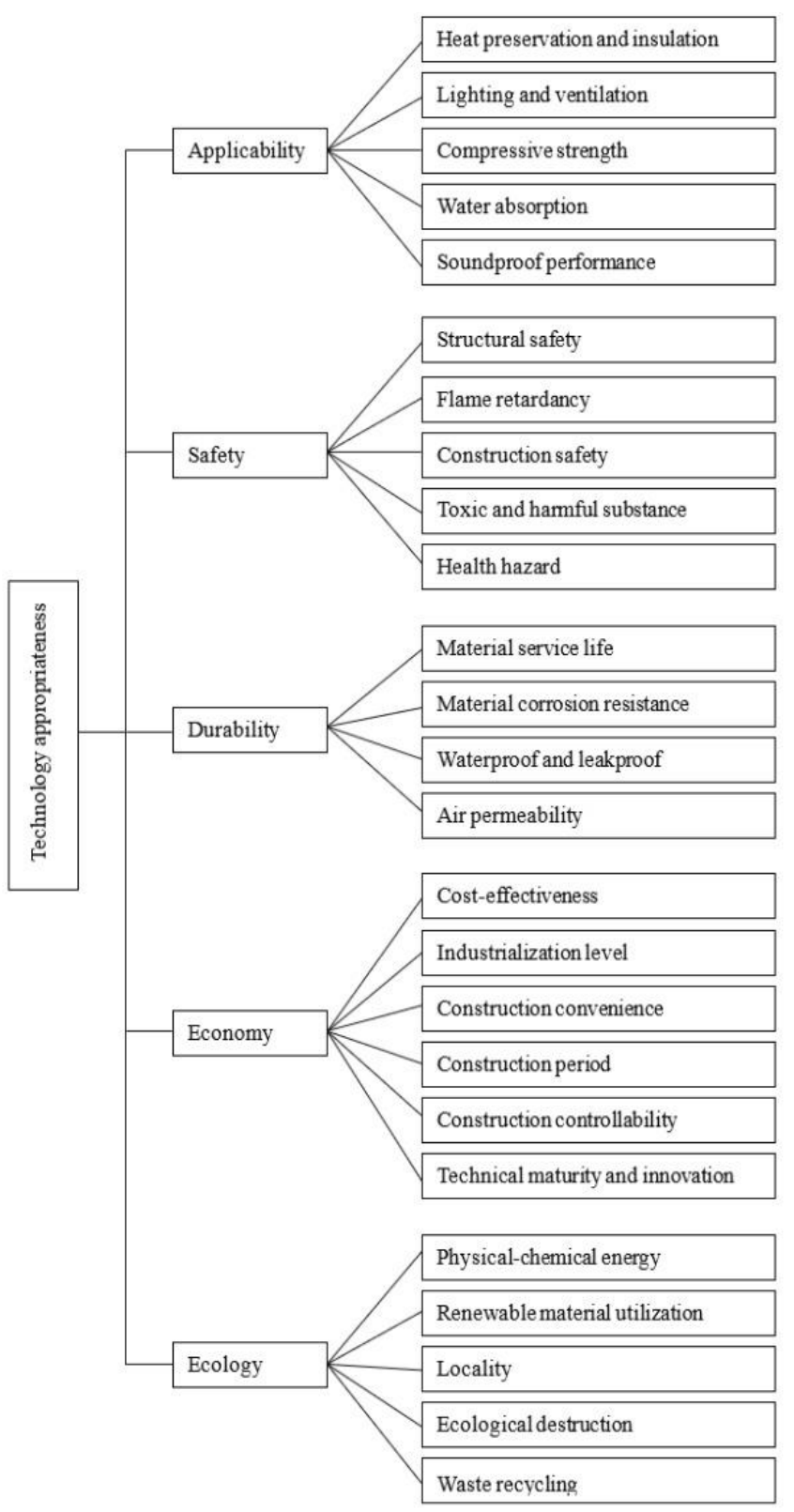

Figure 1. Hierarchy of evaluation indices 
Table 1. Codes of evaluation indices

\begin{tabular}{|c|c|c|c|c|c|}
\hline \multicolumn{2}{|c|}{ Goal A } & \multicolumn{2}{|c|}{ Criterion B } & \multicolumn{2}{|r|}{ Alternative C } \\
\hline Code & Index & Code & Index & Code & Index \\
\hline \multirow{25}{*}{ A01 } & \multirow{25}{*}{$\begin{array}{c}\text { Technology } \\
\text { appropriate } \\
\text { ness }\end{array}$} & \multirow{6}{*}{ B01 } & \multirow{5}{*}{$\begin{array}{l}\text { Applicabilit } \\
y\end{array}$} & $\mathrm{C} 0101$ & Heat preservation and \\
\hline & & & & $\mathrm{C} 0102$ & $\begin{array}{l}\text { 1nsulation } \\
\text { Lighting and } \\
\text { ventilation }\end{array}$ \\
\hline & & & & $\mathrm{C} 0103$ & $\begin{array}{l}\text { Soundproof } \\
\text { performance }\end{array}$ \\
\hline & & & & $\mathrm{C} 0104$ & Compressive strength \\
\hline & & & & $\mathrm{C} 0105$ & Water absorption \\
\hline & & & \multirow{5}{*}{ Safety } & $\mathrm{C} 0201$ & Structural safety \\
\hline & & \multirow{4}{*}{ B02 } & & $\mathrm{C} 0202$ & Flame retardancy \\
\hline & & & & $\mathrm{C} 0203$ & Construction safety \\
\hline & & & & $\mathrm{C} 0204$ & $\begin{array}{l}\text { Toxic and harmful } \\
\text { substance emissions }\end{array}$ \\
\hline & & & & $\mathrm{C} 0205$ & Health hazard \\
\hline & & \multirow{3}{*}{ B03 } & \multirow{4}{*}{ Durability } & $\mathrm{C} 0301$ & Material service life \\
\hline & & & & $\mathrm{C} 0302$ & $\begin{array}{l}\text { Material corrosion } \\
\text { resistance }\end{array}$ \\
\hline & & & & $\mathrm{C} 0303$ & $\begin{array}{l}\text { Waterproof and } \\
\text { leakproof }\end{array}$ \\
\hline & & \multirow{7}{*}{ B04 } & & $\mathrm{C} 0304$ & Air permeability \\
\hline & & & \multirow{6}{*}{ Economy } & $\mathrm{C} 0401$ & Cost-effectiveness \\
\hline & & & & $\mathrm{C} 0402$ & Industrialization level \\
\hline & & & & $\mathrm{C} 0403$ & $\begin{array}{l}\text { Construction } \\
\text { convenience }\end{array}$ \\
\hline & & & & C0404 & Construction period \\
\hline & & & & $\mathrm{C} 0405$ & $\begin{array}{l}\text { Construction } \\
\text { controllability }\end{array}$ \\
\hline & & & & C0406 & $\begin{array}{l}\text { Technical maturity and } \\
\text { innovation }\end{array}$ \\
\hline & & \multirow{5}{*}{ B05 } & \multirow{5}{*}{ Ecology } & $\mathrm{C} 0501$ & $\begin{array}{c}\text { Physical-chemical } \\
\text { energy }\end{array}$ \\
\hline & & & & $\mathrm{C} 0502$ & Renewable material \\
\hline & & & & $\mathrm{C} 0503$ & Locality \\
\hline & & & & $\mathrm{C} 0504$ & Ecological destruction \\
\hline & & & & $\mathrm{C} 0505$ & Waste recycling \\
\hline
\end{tabular}

\subsection{Judgement matrix}

\subsubsection{Questionnaire design}

The relative importance of each criterion and alternative in Figure 1 was evaluated against a 9-point scale (Table 2). For indices on the same level, a pairwise comparison of importance was carried out to produce a judgement matrix, which records the importance of each index on the current layer relative to an index on the superior layer.

Table 2. Saaty's 9-point scale

\begin{tabular}{cc}
\hline Level & Definition (comparison between factors $\mathbf{i}$ and $\mathbf{j}$ ) \\
\hline 1 & Equal relative importance \\
3 & Moderately more important \\
5 & Strongly more important \\
7 & Very strongly more important \\
9 & Extremely more important \\
$2,4,6,8$ & Intermediate values of importance \\
Reciprocal & $\begin{array}{l}\text { The scale value of comparing factor i with factor } \mathrm{j} \\
\text { is the reciprocal of that of comparing factor } \mathrm{j} \text { with } \\
\text { factor } \mathrm{i} .\end{array}$ \\
\hline
\end{tabular}

\subsubsection{Evaluation objects}

This paper attempts to evaluate the technical functions of the energy-saving insulation system for the external envelope of residential buildings. Therefore, the scientific weight of each function was obtained by consulting architects. No weight was assigned to each architect.

\subsubsection{Questionnaire sorting}

Nine building designers were selected from design companies. They were asked to fill out the prepared questionnaire about the weights of technical appropriateness functions for the energy-saving insulation system for the external envelope of residential buildings. A total of 8 replies $(89 \%)$ were recovered.

\subsection{Weight determination and consistency test}

\subsubsection{Index weights and consistency test}

Table 3 shows the judgement matrix, index weights, and consistency ratio (CR).

Table 3. Judgement matrix, index weights, and CRs

\begin{tabular}{ccccccc}
\hline Index & B01 & B02 & B03 & B04 & B05 & Weight Wi \\
\hline B01 & 1 & 6 & 8 & 3 & 5 & 0.498 \\
B02 & $1 / 6$ & 1 & 2 & $1 / 4$ & $1 / 3$ & 0.067 \\
B03 & $1 / 8$ & $1 / 2$ & 1 & $1 / 5$ & $1 / 7$ & 0.040 \\
B04 & $1 / 3$ & 4 & 5 & 1 & $1 / 2$ & 0.181 \\
B05 & $1 / 5$ & 3 & 7 & 2 & 1 & 0.215 \\
& $\lambda_{\max }=5.295 \mathrm{CI}=0.073 \mathrm{RI}=1.12 \mathrm{CR}=0.065$ \\
\hline
\end{tabular}

Note: CI and RI are short for consistency index and randomized index, respectively. The same below.

(1) Each element of judgement matrix $B=\left[b_{i j}\right] 5 \times 5$ is normalized. The general term of the element can be expressed as: $\underset{i j}{b}=\underset{i j}{b} / \sum_{j=1}^{n} \underset{i j}{b}(\mathrm{i}, \mathrm{j}=1,2 \ldots 5)$. Thus, we have:

\begin{tabular}{lllll}
\hline 0.548 & 0.414 & 0.348 & 0.465 & 0.716 \\
0.091 & 0.068 & 0.087 & 0.039 & 0.048 \\
0.068 & 0.035 & 0.044 & 0.031 & 0.021 \\
0.183 & 0.276 & 0.217 & 0.155 & 0.072 \\
0.110 & 0.207 & 0.304 & 0.310 & 0.143 \\
\hline
\end{tabular}

(2) After normalizing each column, the judgement matrices are added up: $\bar{i}=\sum_{j=1}^{n} \bar{b}_{i j}(\mathrm{i}=1,2 \ldots 5)$. Thus, we have: $\bar{W}=(2.491,0.333,0.199,0.903,1.074) \mathrm{T}$.

(3) Vector $\bar{W}$ is normalized to obtain weight $\mathrm{Wi}=\{0.498$, $0.067,0.040,0.181,0.215\}$ in Table $3: \mathrm{Wi}=\{0.498,0.067$, $0.040,0.181,0.215\}$.

(4) Maximum characteristic root

$$
\lambda_{\max }=\sum_{i=1}^{n} \frac{(B W)_{i}}{n W_{i}}
$$

We have $\lambda_{\max }=5.295$.

Since $\mathrm{CI}=\frac{\lambda_{\max }}{n-1}$, we have $\mathrm{CI}=0.073$. By looking up the table, we have $\mathrm{RI}=1.12$. Therefore, $\mathrm{CR}=0.065<0.1$. Hence, the judgement matrix has satisfactory consistency.

\subsubsection{Weight of B01 and consistency test}

Table 4. Judgement matrix, index weights, and CRs of B01

\begin{tabular}{ccccccc}
\hline B01 indices & C0101 & C0102 & & C0103 & C0104 C0105 & Weight Wi \\
\hline $\mathrm{C} 0101$ & 1 & 3 & 7 & 8 & 6 & 0.520 \\
$\mathrm{C} 0102$ & $1 / 3$ & 1 & 4 & 5 & 4 & 0.253 \\
$\mathrm{C} 0103$ & $1 / 7$ & $1 / 4$ & 1 & $1 / 2$ & 2 & 0.073 \\
$\mathrm{C} 0104$ & $1 / 8$ & $1 / 5$ & 2 & 1 & 3 & 0.101 \\
$\mathrm{C} 0105$ & $1 / 6$ & $1 / 4$ & $1 / 2$ & $1 / 3$ & 1 & 0.053 \\
\multicolumn{5}{c}{$\lambda \max =5.320 \mathrm{CI}=0.080 \mathrm{RI}=1.12$} & $\mathrm{CR}=0.071$ \\
\hline
\end{tabular}


Table 4 shows the judgement matrix of index B01 and the corresponding weight and CR.

(1) The elements in each column of judgement matrix $\mathrm{B}=\left[\mathrm{b}_{\mathrm{ij}}\right] 5 \times 5$ are normalized. The general term of the element can be expressed as: $\underset{i j}{b}=\underset{i j}{b} / \sum_{j=1}^{n} \underset{i j}{b}(\mathrm{i}, \mathrm{j}=1,2 \ldots 5)$. Thus, we have:

\begin{tabular}{llllc}
\hline 0.565 & 0.638 & 0.483 & 0.539 & 0.374 \\
0.189 & 0.213 & 0.276 & 0.337 & 0.25 \\
0.081 & 0.053 & 0.069 & 0.035 & 0.125 \\
0.071 & 0.043 & 0.138 & 0.067 & 0.188 \\
0.094 & 0.053 & 0.034 & 0.022 & 0.063 \\
\hline
\end{tabular}

(2) After normalizing each column, the judgement matrices are added up: $\bar{i}=\sum_{j=1}^{n}{ }_{i j}(\mathrm{i}=1,2 \ldots 5)$. Thus, we have: $\bar{W}=(2.599,1.265,0.363,0.507,0.266) \mathrm{T}$.

(3) Vector $\bar{W}$ is normalized to obtain weights: $\mathrm{Wi}=\{0.498$, $0.067,0.040,0.181,0.215\}$.

(4) Maximum characteristic root

$$
\lambda_{\max }=\sum_{i=1}^{n} \frac{(B W)_{i}}{n W_{i}}
$$

We have $\lambda_{\max }=5.320$.

Since $\mathrm{CI}=\frac{\lambda_{\max }-n}{n-1}$, we have $\mathrm{CI}=0.080$. By looking up the table, we have $\mathrm{RI}=1.12$. Therefore, $\mathrm{CR}=0.071<0.1$. Hence, the judgement matrix has satisfactory consistency.

\subsubsection{Weight of B02 and consistency test}

Table 5 shows the judgement matrix of index B02 and the corresponding weight and CR.

Table 5. Judgement matrix, index weights, and CRs of B02

\begin{tabular}{ccccccc}
\hline B02 indices & C0201 & C0202 & C0203 & C0204 & C0205 & Weight Wi \\
\hline $\mathrm{C} 0201$ & 1 & $1 / 4$ & 2 & 2 & $1 / 2$ & 0.136 \\
$\mathrm{C} 0202$ & 4 & 1 & 5 & 5 & 3 & 0.484 \\
$\mathrm{C} 0203$ & $1 / 2$ & $1 / 5$ & 1 & 1 & $1 / 3$ & 0.079 \\
$\mathrm{C} 0204$ & $1 / 2$ & $1 / 5$ & 1 & 1 & $1 / 3$ & 0.079 \\
$\mathrm{C} 0205$ & 2 & $1 / 3$ & 3 & 3 & 1 & 0.222 \\
& $\lambda \max =5.059 \mathrm{CI}=0.015 \mathrm{RI}=1.12 \mathrm{CR}=0.013$ \\
\hline
\end{tabular}

(1) The elements in each column of judgement matrix $\mathrm{B}=\left[\mathrm{b}_{\mathrm{ij}}\right] 5 \times 5$ are normalized. The general term of the element can be expressed as: $\underset{i j}{b}=\underset{i j}{b} / \sum_{j=1}^{n} \underset{i j}{b}(\mathrm{i}, \mathrm{j}=1,2 \ldots 5)$. Thus, we have:

\begin{tabular}{ccccc}
\hline 0.125 & 0.126 & 0.167 & 0.167 & 0.097 \\
0.5 & 0.504 & 0.417 & 0.417 & 0.581 \\
0.0625 & 0.101 & 0.083 & 0.083 & 0.064 \\
0.0625 & 0.101 & 0.083 & 0.083 & 0.064 \\
0.25 & 0.168 & 0.25 & 0.25 & 0.194 \\
\hline
\end{tabular}

(2) After normalizing each column, the judgement matrices are added up: $\bar{W}=\sum_{j=1}^{n}{ }_{i j} \bar{i}(\mathrm{i}=1,2 \ldots 5)$. Thus, we have: $\bar{W}=(0.682,2.419,0.394,0.394,1.112) \mathrm{T}$.

(3) Vector $\bar{W}$ is normalized to obtain weights: $\mathrm{Wi}=\{0.136$, $0.484,0.079,0.079,0.222\}$.

(4) Maximum characteristic root

$$
\lambda_{\max }=\sum_{i=1}^{n} \frac{(B W)_{i}}{n W_{i}}
$$

We have $\lambda_{\max }=5.059$.

Since $\mathrm{CI}=\frac{\lambda_{\max }-n}{n-1}$, we have $\mathrm{CI}=0.015$. By looking up the table, we have $\mathrm{RI}=1.12$. Therefore, $\mathrm{CR}=0.013<0.1$. Hence, the judgement matrix has satisfactory consistency.

\subsubsection{Weight of B03 and consistency test}

Table 6 shows the judgement matrix of index B03 and the corresponding weight and CR.

Table 6. Judgement matrix, index weights, and CRs of B03

\begin{tabular}{cccccc}
\hline B03 indices & C0301 & C0302 & C0303 & C0304 & Weight Wi \\
\hline $\mathrm{C} 0301$ & 1 & 6 & 5 & 6 & 0.642 \\
$\mathrm{C} 0302$ & $1 / 6$ & 1 & $1 / 2$ & 1 & 0.095 \\
$\mathrm{C} 0303$ & $1 / 5$ & 2 & 1 & 2 & 0.168 \\
$\mathrm{C} 0304$ & $1 / 6$ & 1 & $1 / 2$ & 1 & 0.095 \\
$\lambda \max =4.033$ & $\mathrm{CI}=0.011$ & $\mathrm{RI}=0.90$ & $\mathrm{CR}=0.012$ \\
\hline
\end{tabular}

(1) The elements in each column of judgement matrix $\mathrm{B}=\left[\mathrm{b}_{\mathrm{ij}}\right] 5 \times 5$ are normalized. The general term of the element can be expressed as: $\underset{i j}{b}=\underset{i j}{b} / \sum_{j=1}^{n} \underset{i j}{b}(\mathrm{i}, \mathrm{j}=1,2 \ldots 4)$. Thus, we have:

\begin{tabular}{llll}
\hline 0.652 & 0.6 & 0.714 & 0.6 \\
0.109 & 0.1 & 0.071 & 0.1 \\
0.130 & 0.2 & 0.144 & 0.2 \\
0.109 & 0.1 & 0.071 & 0.1 \\
\hline
\end{tabular}

(2) After normalizing each column, the judgement matrices are added up: $\bar{i}=\sum_{j=1}^{n}{ }_{i j} \bar{b}(\mathrm{i}=1,2 \ldots 4)$. Thus, we have: $\bar{W}=(2.566,0.38,0.674,0.38) \mathrm{T}$.

(3) Vector $\bar{W}$ is normalized to obtain weights: $\mathrm{Wi}=\{0.642$, $0.095,0.168,0.095\}$.

(4) Maximum characteristic root

$$
\lambda_{\max }=\sum_{i=1}^{n} \frac{(B W)_{i}}{n W_{i}}
$$

We have $\lambda_{\max }=4.033$.

Since $\mathrm{CI}=\frac{\lambda_{\max }-n}{n-1}$, we have $\mathrm{CI}=0.011$. By looking up the table, we have $\mathrm{RI}=0.90$. Therefore, $\mathrm{CR}=0.012<0.1$. Hence, the judgement matrix has satisfactory consistency.

\subsubsection{Weight of B04 and consistency test}

Table 7 shows the judgement matrix of index B04 and the corresponding weight and CR.

Table 7. Judgement matrix, index weights, and CRs of B04

\begin{tabular}{cccccccc}
\hline B04 indices & C0401 & C402 & C0403 C0404 C0405 & C406 & Weight Wi \\
\hline $\mathrm{C} 0401$ & 1 & 4 & 7 & 7 & 4 & 6 & 0.469 \\
$\mathrm{C} 0402$ & $1 / 4$ & 1 & 4 & 3 & 1 & 3 & 0.172 \\
$\mathrm{C} 0403$ & $1 / 7$ & $1 / 4$ & 1 & 1 & $1 / 4$ & $1 / 2$ & 0.049 \\
$\mathrm{C} 0404$ & $1 / 7$ & $1 / 3$ & 1 & 1 & $1 / 3$ & $1 / 2$ & 0.052 \\
$\mathrm{C} 0405$ & $1 / 4$ & 1 & 4 & 3 & 1 & $1 / 3$ & 0.132 \\
$\mathrm{C} 0406$ & $1 / 6$ & $1 / 3$ & 2 & 2 & 3 & 1 & 0.126 \\
& $\lambda \max =6.539$ & $\mathrm{CI}=0.1 \mathrm{RI}=1.24 \mathrm{CR}=0.087$ & \\
\hline
\end{tabular}


(1) The elements in each column of judgement matrix $\mathrm{B}=\left[\mathrm{b}_{\mathrm{ij}}\right] 5 \times 5$ are normalized. The general term of the element can be expressed as: $\underset{i j}{b}=\underset{i j}{b} / \sum_{j=1}^{n} \underset{i j}{b}(\mathrm{i}, \mathrm{j}=1,2 \ldots 6)$. Thus, we have:

\begin{tabular}{llllll}
\hline 0.512 & 0.578 & 0.367 & 0.412 & 0.417 & 0.469 \\
0.128 & 0.145 & 0.211 & 0.176 & 0.104 & 0.172 \\
0.073 & 0.036 & 0.053 & 0.059 & 0.027 & 0.049 \\
0.073 & 0.048 & 0.053 & 0.059 & 0.035 & 0.052 \\
0.128 & 0.145 & 0.211 & 0.176 & 0.104 & 0.132 \\
0.086 & 0.048 & 0.105 & 0.118 & 0.313 & 0.126 \\
\hline
\end{tabular}

(2) After normalizing each column, the judgement matrices are added up: $\bar{i}=\sum_{j=1}^{n} \bar{b}_{i j}(\mathrm{i}=1,2 \ldots 6)$. Thus, we have: $\bar{W}=(2.815,1.03,0.292,0.312,0.793,0.758) \mathrm{T}$.

(3) Vector $\bar{W}$ is normalized to obtain weights: $\mathrm{Wi}=\{0.469$, $0.172,0.049,0.052,0.132,0.126\}$.

(4) Maximum characteristic root

$$
\lambda_{\max }=\sum_{i=1}^{n} \frac{(B W)_{i}}{n W_{i}}
$$

We have $\lambda_{\text {max }}=6.539$.

Since $\mathrm{CI}=\frac{\lambda_{\max }-n}{n-1}$, we have $\mathrm{CI}=0.100$. By looking up the table, we have $\mathrm{RI}=1.24$. Therefore, $\mathrm{CR}=0.087<0.1$. Hence, the judgement matrix has satisfactory consistency.

\subsubsection{Weight of B05 and consistency test}

Table 8 shows the judgement matrix of index B05 and the corresponding weight and $\mathrm{CR}$.

Table 8. Judgement matrix, index weights, and CRs of B05

\begin{tabular}{ccccccc}
\hline \multicolumn{1}{c}{ B05 indicesC0501C0502C0503C0504C0505Weight Wi } \\
\hline $\mathrm{C} 0501$ & 1 & 1 & $1 / 3$ & 1 & $1 / 2$ & 0.120 \\
$\mathrm{C} 0502$ & 1 & 1 & $1 / 3$ & 1 & $1 / 2$ & 0.120 \\
$\mathrm{C} 0503$ & 3 & 3 & 1 & 3 & 4 & 0.437 \\
$\mathrm{C} 0504$ & 1 & 1 & $1 / 3$ & 1 & $1 / 2$ & 0.120 \\
$\mathrm{C} 0505$ & 2 & 2 & $1 / 4$ & 2 & 1 & 0.203 \\
\multicolumn{5}{c}{$\lambda$ max $=4.12$} & $\mathrm{CI}=0.04 \mathrm{RI}=0.9$ & $\mathrm{CR}=0.044$ \\
\hline
\end{tabular}

(1) The elements in each column of judgement matrix $\mathrm{B}=\left[\mathrm{b}_{\mathrm{ij}}\right] 5 \times 5$ are normalized. The general term of the element can be expressed as: $\underset{i j}{b}=\underset{i j}{b} / \sum_{j=1}^{n} \underset{i j}{b}(\mathrm{i}, \mathrm{j}=1,2 \ldots 5)$. Thus, we have:

\begin{tabular}{lllll}
\hline 0.125 & 0.125 & 0.148 & 0.125 & 0.077 \\
0.125 & 0.125 & 0.148 & 0.125 & 0.077 \\
0.375 & 0.375 & 0.444 & 0.375 & 0.615 \\
0.125 & 0.125 & 0.148 & 0.125 & 0.077 \\
0.250 & 0.250 & 0.112 & 0.250 & 0.154 \\
\hline
\end{tabular}

(2) After normalizing each column, the judgement matrices are added up: $\bar{i}=\sum_{j=1}^{n}{ }_{i j} \bar{b}(\mathrm{i}=1,2 \ldots 5)$. Thus, we have: $\bar{W}=(0.600,0.600,2.184,0.600,1.016) \mathrm{T}$.

(3) Vector $\bar{W}$ is normalized to obtain weights: $\mathrm{Wi}=\{0.120$, $0.120,0.437,0.120,0.203\}$.

(4) Maximum characteristic root

$$
\lambda_{\max }=\sum_{i=1}^{n} \frac{(B W)_{i}}{n W_{i}}
$$

We have $\lambda_{\max }=4.12$.

Since $\mathrm{CI}=\frac{\lambda_{\max }-n}{n-1}$, we have $\mathrm{CI}=0.04$. By looking up the table, we have $\mathrm{RI}=0.90$. Therefore, $\mathrm{CR}=0.044<0.1$. Hence, the judgement matrix has satisfactory consistency.

\section{CASE ANALYSIS}

\subsection{Project background and relevant parameters}

The above evaluation method was adopted to analyze four insulation schemes of residential buildings, denoted as A-D in turn. Figure 2 shows the floor plan of the residential building, and Tables 9 and 10 show the overview of the engineering project.

(1) Scheme A

Table 11 shows the energy-saving insulation system of the envelope structure in scheme A. Table 12 reports the designed energy-saving rate of the building.

(2) Scheme B

Table 13 shows the energy-saving insulation system of the envelope structure in scheme B. Table 14 reports the designed energy-saving rate of the building.

(3) Scheme C

Table 15 shows the energy-saving insulation system of the envelope structure in scheme C. Table 16 reports the designed energy-saving rate of the building.

(4) Scheme D

Table 17 shows the energy-saving insulation system of the envelope structure in scheme D. Table 18 reports the designed energy-saving rate of the building.

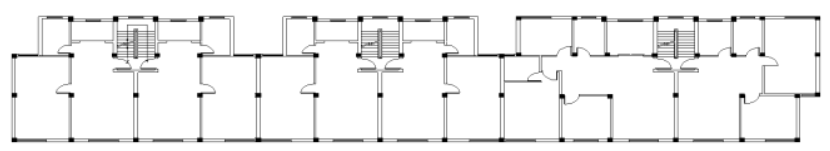

Figure 2. Floor plan of residential building 20\#

\begin{tabular}{|c|c|c|c|c|c|}
\hline Project name & $\begin{array}{c}\text { Residential buildings 16\# and 17\# of a residential commt } \\
\text { Chuzhou, Anhui Province, China }\end{array}$ & iity in & Project locatio & Chuz & $\begin{array}{l}\text {, Anhui Province, } \\
\text { China }\end{array}$ \\
\hline $\begin{array}{l}\text { Construction } \\
\text { company }\end{array}$ & A property development company in Chuzhou & & Floor area $(\mathrm{A} 0$ & & $3528 \mathrm{~m}^{2}$ \\
\hline Building shape & Strip shape & $\begin{array}{l}\text { Shape } \\
\text { coefficient }\end{array}$ & 0.31 & Surface area & $3313.06 \mathrm{~m}^{2}$ \\
\hline Number of floors & 6 & $\begin{array}{c}\text { Building } \\
\text { height }\end{array}$ & $18.050 \mathrm{~m}$ & $\begin{array}{l}\text { Building } \\
\text { volume }\end{array}$ & $10140.96 \mathrm{~m}^{3}$ \\
\hline
\end{tabular}

Table 9. Project overview 
Table 10. Surface area of envelope components

\begin{tabular}{cccccc}
\hline Type of structure & Walls & Columns & Beams & External windows and doors & Roof \\
\hline Area $\left(\mathbf{m}^{\mathbf{2}}\right)$ & 1280.62 & 205.77 & 294.77 & 555.57 & 553.32 \\
\hline
\end{tabular}

Table 11. Energy-saving insulation system of the envelope structure

\begin{tabular}{c|c} 
Wall & $\begin{array}{c}\text { Alkali resistant broken fiber gridding cloth, anti-crack mortar }(5 \mathrm{~mm}) \text {-rubber powder polystyrene particle insulation } \\
\text { mortar }(20 \mathrm{~mm})-240 \mathrm{~mm} \text { porous clay brick-composite mortar 20 mm }\end{array}$ \\
$\begin{array}{c}\text { Flat roof } \\
\text { Floor slab } \\
\text { Doors }\end{array}$ & $\begin{array}{c}\text { Blocks-coarse sand cushion }(25 \mathrm{~mm}) \text {-polymer modified asphalt waterproofing membrane }(4 \mathrm{~mm}) \text {-cement mortar } 1(20 \\
\text { mm }) \text {-extruded polystyrene board }(20 \mathrm{~mm}) \text {-light-weight aggregate concrete }(30 \mathrm{~mm}) \text {-reinforced concrete }(100 \mathrm{~mm})\end{array}$ \\
$\begin{array}{c}\text { Windows } \\
\text { Cement mortar floor, raised floor slab with natural ventilation at bottom } \\
\text { Energy-saving external door }\end{array}$ \\
$\begin{array}{c}\text { Thermal bridge } \\
\text { columns and beams }\end{array}$ & $\begin{array}{c}\text { Alkali resistant broken fiber gridding cloth, anti-crack mortar }(5 \mathrm{~mm}) \text {-rubber powder polystyrene particle insulation } \\
\text { mortar }(20 \mathrm{~mm}) \text {-reinforced concrete }(240 \mathrm{~mm}) \text {-composite mortar } 20 \mathrm{~mm}\end{array}$ \\
\hline
\end{tabular}

Table 12. Designed building energy-saving rate

\begin{tabular}{cccc}
\hline Calculated result & Designed building & Reference building & Energy-saving rate \\
\hline Annual power consumption $\left(\mathbf{k W h} / \mathbf{m}^{\mathbf{2}}\right)$ & 46.38 & 48.63 & $52.31 \%$ \\
\hline
\end{tabular}

Table 13. Energy-saving insulation system of the envelope structure

\begin{tabular}{|c|c|}
\hline Wall & $\begin{array}{c}\text { Alkali resistant broken fiber gridding cloth, anti-crack mortar }(5 \mathrm{~mm}) \text {-polystyrene board (30 } \mathrm{mm})-240 \mathrm{~mm} \text { porous clay } \\
\text { brick-composite mortar } 20 \mathrm{~mm}\end{array}$ \\
\hline & $\begin{array}{l}\text { Blocks-coarse sand cushion }(25 \mathrm{~mm}) \text {-polymer modified asphalt waterproofing membrane }(4 \mathrm{~mm}) \text {-cement mortar } 1(20 \\
\mathrm{mm}) \text {-extruded polystyrene board }(30 \mathrm{~mm}) \text {-light-weight aggregate concrete }(30 \mathrm{~mm}) \text {-reinforced concrete }(100 \mathrm{~mm})\end{array}$ \\
\hline $\begin{array}{c}\text { Floor slab } \\
\text { Doors }\end{array}$ & $\begin{array}{l}\text { Cement mortar floor, raised floor slab with natural ventilation at bottom } \\
\text { Energy-saving external door }\end{array}$ \\
\hline Windows & Plastic steel single frame ordinary hollow glass window, with a heat transfer coefficient of $2.5 \mathrm{~W} /\left(\mathrm{m}^{2} \cdot \mathrm{K}\right)$ \\
\hline $\begin{array}{l}\text { Thermal bridge } \\
\text { columns and beams }\end{array}$ & $\begin{array}{l}\text { Alkali resistant broken fiber gridding cloth, anti-crack mortar }(5 \mathrm{~mm}) \text {-rubber powder polystyrene particle insulation } \\
\text { mortar }(30 \mathrm{~mm}) \text {-reinforced concrete }(240 \mathrm{~mm}) \text {-composite mortar } 20 \mathrm{~mm}\end{array}$ \\
\hline
\end{tabular}

Table 14. Designed building energy-saving rate

\begin{tabular}{cccc}
\hline Calculated result & Designed building & Reference building & Energy-saving rate \\
\hline Annual power consumption $\left(\mathbf{k W h} / \mathbf{m}^{\mathbf{2}}\right)$ & 42.53 & 48.63 & $56.3 \%$ \\
\hline
\end{tabular}

Table 15. Energy-saving insulation system of the envelope structure

\begin{tabular}{cc}
\hline Wall & Alkali resistant broken fiber gridding cloth, anti-crack mortar $(5 \mathrm{~mm})-240 \mathrm{~mm}$ autoclaved lightweight aerated concrete- \\
composite mortar $20 \mathrm{~mm}$
\end{tabular}

Table 16. Designed building energy-saving rate

\begin{tabular}{cccc}
\hline Calculated result & Designed building & Reference building & Energy-saving rate \\
\hline Annual power consumption $\left(\mathbf{k W h} / \mathbf{m}^{\mathbf{2}}\right)$ & 42.2 & 48.63 & $56.6 \%$ \\
\hline
\end{tabular}

Table 17. Energy-saving insulation system of the envelope structure

\begin{tabular}{|c|c|}
\hline Wall & $\begin{array}{c}\text { Alkali resistant broken fiber gridding cloth, anti-crack mortar }(5 \mathrm{~mm}) \text {-rigid foam polyurethane board }(50 \mathrm{~mm})-240 \mathrm{~mm} \\
\text { porous clay brick-composite mortar } 20 \mathrm{~mm}\end{array}$ \\
\hline & $\begin{array}{l}\text { Blocks-coarse sand cushion }(25 \mathrm{~mm}) \text {-polymer modified asphalt waterproofing membrane }(4 \mathrm{~mm}) \text {-cement mortar } 1(20 \\
\mathrm{mm}) \text {-extruded polystyrene board }(50 \mathrm{~mm}) \text {-light-weight aggregate concrete }(30 \mathrm{~mm}) \text {-reinforced concrete }(100 \mathrm{~mm})\end{array}$ \\
\hline Floor slab & Cement mortar floor, raised floor slab with natural ventilation at bottom \\
\hline Doors & Energy-saving external door \\
\hline Windows & Plastic steel single frame low radiation hollow glass window, with a heat transfer coefficient of $1.8 \mathrm{~W} /\left(\mathrm{m}^{2} \cdot \mathrm{K}\right)$ \\
\hline $\begin{array}{l}\text { Thermal bridge } \\
\text { columns and beams }\end{array}$ & $\begin{array}{l}\text { Alkali resistant broken fiber gridding cloth, anti-crack mortar }(5 \mathrm{~mm}) \text {-rigid foam polyurethane board }(50 \mathrm{~mm}) \text { - } \\
\text { reinforced concrete }(240 \mathrm{~mm}) \text {-composite mortar } 20 \mathrm{~mm}\end{array}$ \\
\hline
\end{tabular}


Table 18. Designed building energy-saving rate

\begin{tabular}{c|ccc}
\hline Calculated result & Designed building & Reference building & Energy-saving rate \\
\hline Annual power consumption $\left(\mathbf{k W h} / \mathbf{m}^{\mathbf{2}}\right)$ & 34.91 & 48.63 & $64.1 \%$ \\
\hline
\end{tabular}

\section{VALUE ANALYSIS}

\subsection{Index comparison}

(1) Applicability

According to the matrix of index weights in Table 4, the applicability indices are ranked in descending order of weight as heat preservation and insulation, lighting and ventilation, compressive strength, soundproof performance, and water absorption. As a basic attribute of energy-saving insulation system, heat preservation and insulation has a much greater weigh than the other indices. Thus, the performance of heat preservation and insulation is the main indicator of scheme appropriateness. In terms of energy-saving rate, Scheme D had an energy-saving rate of $64.1 \%$, achieving the best heat preservation and insulation performance. Schemes B and C were comparable in heat preservation and insulation. Both achieved an energy-saving rate of $56 \%$. Scheme A performed the worst in this respect.

In terms of lighting and ventilation, Scheme $\mathrm{D}$ was not as good as the other schemes, because the low radiation LOW-E glass window cannot be open for a long time. The soundproof performance should be considered comprehensively in the light of wall insulation material and door/window material. Schemes B and D had an obvious advantage in soundproof performance than Schemes A and C. The reason is that both adopt composite insulation of board walls, and use plastic steel and low radiation hollow glasses.

The compressive strength and water absorption are mainly dependent on the attributes of wall insulation material in the energy-saving insulation system. Compressive strength directly reflects the material strength, and the resistance of material to external damage. Among the wall insulation materials, the insulation material of the aerated concrete wall boasts the highest compressive strength, while that of polystyrene foam plastic board has the lowest compressive strength. Thus, the latter is not suitable for positions that are often accessed by humans.

Water absorption is also determined by the attributes of wall insulation material. The value of the index is negatively correlated with material insulation capacity. The stronger the water absorption, the higher the heat transfer coefficient, and the lower the energy-saving rate. The water absorption performance was measured by the modified thermal conductivity coefficient. The larger the coefficient, the stronger the water absorption of the material in the environment. The modified coefficient of each material was looked up in Standard for Energy-Saving Design of Residential Buildings in Anhui Province. The aerated concrete wall has the highest modified coefficient, and thus the largest water absorption, followed by rigid foam polyurethane board, polystyrene board, and rubber powder polystyrene particles.

(2) Safety

Recently, the combustion of insulation materials has induced frequent building fires across China. In consequence, the flame retardancy of the insulation system is given high priority. According to the matrix of index weights in Table 5, the safety indices are ranked in descending order of weight as flame retardancy, health hazard, structural safety, construction safety, and toxic and harmful substance emissions. As discussed in the previous sections, Scheme $\mathrm{C}$ had an obvious advantage in safety, because it uses self-insulating material in most walls, while the other schemes differed slightly in safety.

(3) Durability

According to the matrix of index weights in Table 6, material service life is the most important durability index. If the material service life is inconsistent with the lifecycle of the building, the material needs to be removed before the building reaches its age limit, resulting in lots of white waste. The ensuing retrofitting cost and project are another thorny issue. Scheme $\mathrm{C}$ had an obvious advantage in durability, because its energy-saving insulation system has the same service life as the building.

(4) Economy

According to the matrix of index weights in Table 7, the economy indices are ranked in descending order of weight as cost-effectiveness, industrialization level, construction period, construction convenience, construction controllability, and technical maturity and innovation. For doors and windows, as mentioned in Section 4, plastic steel hollow glass window is the most cost effective choice, without sacrificing the beauty of the façade. For wall insulation, the self-insulating material of walls is economic, and technically feasible, capable of realizing a high energy-saving rate. As a result, Scheme C boasts the highest cost effectiveness, followed in turn by Scheme B. Despite having the highest energy-saving rate, Scheme D had the lowest cost effectiveness, due to the high cost-input ratio of its energy-saving insulation system. The other economic indies are related to the construction process of the energy-saving insulation system. Scheme $\mathrm{C}$ enjoys high construction convenience and construction controllability, which are brought by relatively high industrialization level. The high industrialization level also helps greatly shorten the construction period. Besides, the high technical maturity and innovation significantly reduces the payback period. That is why Scheme C is the optimal choice in all respects. Scheme A was slightly better than Schemes B and D. The reason is that the composite insulation of board walls in Schemes B and D, plus the LOW-E low radiation window of Scheme D, require sophisticated construction skills.

\section{(5) Ecology}

According to the matrix of index weights in Table 8, the ecology indices are ranked in descending order of weight as locality, waste recycling, physical-chemical energy, renewable material, and ecological destruction. Schemes A, B, and D were outshined in all respects by Scheme $\mathrm{C}$, for the composite wall insulation material is poorer than the self-insulating material. In terms of energy-saving windows, aluminum alloy window is slightly better than plastic steel window in ecology. Overall, Scheme $\mathrm{C}$ had an obvious advantage in ecology.

\subsection{Functional coefficients of different schemes}

The scores of each scheme are listed in Table 19.

The weighted functional scores of the four schemes are listed in Table 20 
Table 19. Codes of evaluation index system

\begin{tabular}{|c|c|c|c|c|c|c|c|c|}
\hline \multicolumn{5}{|c|}{ Goal ACriterion BAlternative C } & \multicolumn{4}{|c|}{ Score } \\
\hline Code & \multicolumn{3}{|c|}{ CodeWeight Code } & Weight & $\begin{array}{r}\text { Scher } \\
\text { A }\end{array}$ & \multirow{2}{*}{$\begin{array}{c}\text { chen } \\
\text { B }\end{array}$} & \multirow{2}{*}{$\begin{array}{l}\text { cher } \\
\text { C }\end{array}$} & $\begin{array}{c}\text { chen } \\
\text { D }\end{array}$ \\
\hline \multirow{25}{*}{ A01 } & \multirow{5}{*}{\multicolumn{2}{|c|}{ B01 0.498}} & $\mathrm{C} 0101$ & 0.520 & 7 & & & 9 \\
\hline & & & $\mathrm{C} 0102$ & 0.253 & 8 & 8 & 8 & 7.5 \\
\hline & & & $\mathrm{C} 0103$ & 0.073 & 7.5 & 8 & 7 & 8 \\
\hline & & & $\mathrm{C} 0104$ & 0.101 & 8 & 7 & 9 & 7 \\
\hline & & & $\mathrm{C} 0105$ & 0.053 & 5 & 6 & 8 & 7 \\
\hline & \multirow{5}{*}{ B02 } & & $\mathrm{C} 0201$ & 0.136 & 8 & 7 & 9 & 7 \\
\hline & & \multirow{4}{*}{0.067} & $\mathrm{C} 0202$ & 0.484 & 8 & 7 & 9 & 7 \\
\hline & & & $\mathrm{C} 0203$ & 0.079 & 7.5 & 7 & 8 & 7 \\
\hline & & & $\mathrm{C} 0204$ & 0.079 & 7 & 6 & 8 & 6 \\
\hline & & & $\mathrm{C} 0205$ & 0.222 & 7 & 6 & 8 & 6 \\
\hline & \multirow{4}{*}{\multicolumn{2}{|c|}{ В03 0.040}} & $\mathrm{C} 0301$ & 0.642 & 7 & 7 & 9 & 7 \\
\hline & & & $\mathrm{C} 0302$ & 0.095 & 6 & 7 & 8 & 7 \\
\hline & & & $\mathrm{C} 0303$ & 0.168 & 7 & 7 & 8 & 7 \\
\hline & & & $\mathrm{C} 0304$ & 0.095 & 5 & 7 & 6 & 8 \\
\hline & \multirow{6}{*}{\multicolumn{2}{|c|}{ B04 0.181}} & $\mathrm{C} 0401$ & 0.469 & 7 & 8 & 9 & 6 \\
\hline & & & $\mathrm{C} 0402$ & 0.172 & 7 & 7 & 8 & 7 \\
\hline & & & $\mathrm{C} 0403$ & 0.049 & 8 & 7 & 9 & 7 \\
\hline & & & $\mathrm{C} 0404$ & 0.052 & 8 & 8 & 9 & 8 \\
\hline & & & $\mathrm{C} 0405$ & 0.132 & 8 & 7 & 9 & 7 \\
\hline & & & $\mathrm{C} 0406$ & 0.126 & 7 & 7 & 8 & 7 \\
\hline & \multirow{5}{*}{ B05 } & & $\mathrm{C} 0501$ & 0.120 & 7 & 6.5 & 8 & 6 \\
\hline & & & $\mathrm{C} 0502$ & 0.120 & 6 & 7 & 8 & 7 \\
\hline & & 0.215 & $\mathrm{C} 0503$ & 0.437 & 6 & 7 & 7 & 7 \\
\hline & & & $\mathrm{C} 0504$ & 0.120 & 6 & 7 & 8 & 7 \\
\hline & & & $\mathrm{C} 0505$ & 0.203 & 6 & 7 & 8 & 7 \\
\hline
\end{tabular}

Table 20. Weighted functional scores of the four schemes

\begin{tabular}{lcccccc}
\hline \multicolumn{7}{c}{ ApplicabilitySafetyDurabilityEconomyEcologyTotal } \\
\hline Scheme A & 3.628 & 0.513 & 0.269 & 1.309 & 1.316 & 7.035 \\
Scheme B & 3.881 & 0.449 & 0.28 & 1.361 & 1.492 & 7.463 \\
Scheme C & 3.998 & 0.578 & 0.284 & 1.575 & 1.626 & 8.061 \\
Scheme D & 4.103 & 0.449 & 0.338 & 1.192 & 1.479 & 7.561 \\
\hline
\end{tabular}

The results show that $\mathrm{FC}>\mathrm{FD}>\mathrm{FB}>\mathrm{FA}$. Figure 3 compares top-rated Scheme $\mathrm{C}$ with the lowest-rated Scheme A.

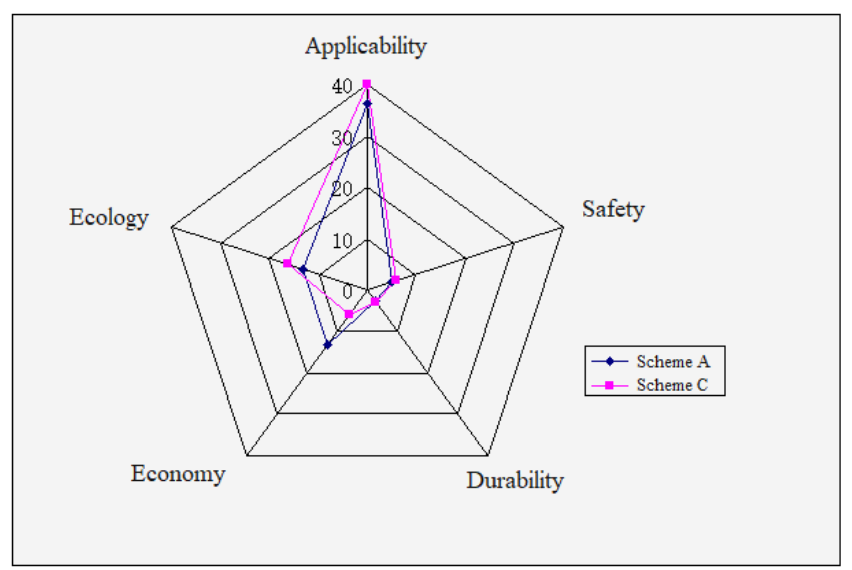

Figure 3. Comparison of criterion scores of the four schemes

The score of Scheme A can be calculated by:

$$
\begin{aligned}
& F A=\frac{\text { Weighted functional score of scheme } A}{\text { Weighted total functional score of the four schemes }} \\
& =\frac{7.035}{7.035+7.463+8.061+7.561}=0.234
\end{aligned}
$$

Similarly, it can be obtained that $\mathrm{FB}=0.248, \mathrm{FC}=0.268$, and $\mathrm{FD}=0.251$.

\subsection{Cost coefficients}

The cost per unit of floor area was calculated for each scheme. The energy-saving insulation cost per square meters of Scheme A was 123.351 yuan, that of Scheme B was 104.947 yuan, that of Scheme C was 107.923 yuan, and that of Scheme $\mathrm{D}$ was 182.274 yuan. The cost coefficient CA of Scheme A can be calculated by:

$$
\begin{aligned}
& C A=\frac{\text { Cost of Scheme } A}{\text { Total cost of all schemes }} \\
& =\frac{123.351}{123.351+104.947+107.923+182.274}=0.238
\end{aligned}
$$

Similarly, it can be obtained that $\mathrm{CB}=0.202, \mathrm{CC}=0.208$, and $\mathrm{CD}=0.352$.

\subsection{Value coefficients}

If value coefficient $\mathrm{V}$ is equal to 1 , then the selected energysaving insulation system is basically appropriate.

If value coefficient $\mathrm{V}$ is greater than 1 , then the selected energy-saving insulation system is highly appropriate, and should be preferred.

If value coefficient $\mathrm{V}$ is smaller than 1 , then the selected energy-saving insulation system is inappropriate, and should be modified.

The value coefficient VA of Scheme A can be calculated by:

$$
V A=\frac{F A}{C A}=\frac{0.234}{0.238}=0.983
$$

Similarly, it can be obtained that $\mathrm{VB}=1.227, \mathrm{VC}=1.288$, and $\mathrm{VD}=0.713$.

The value coefficients of Schemes B and C were both greater than 1 , indicating that the energy-saving rate was around $56 \%$. It is appropriate to choose the two schemes for energy-saving insulation of the external envelope structure. Since VC $>$ VB, VC is the most appropriate scheme. The value coefficient of Scheme A was close to 1 , indicating that the scheme is not very appropriate, and should be modified. The value coefficient of Scheme D was smaller than 1, indicating that the energy-saving rate was around $65 \%$. It is inappropriate to adopt this energy-saving insulation system for the external envelope structure. This means the appropriateness of energysaving insulation system cannot be evaluated scientifically based on technical indices alone. The quality of a scheme must be judged through overall consideration of various aspects.

\subsection{Improvement suggestions}

Admittedly, Scheme A is superior in safety, durability, economy, and ecology, and low in cost input. But the energysaving standard of the scheme is low. The defects of Scheme A concentrate in applicability. That is why the value coefficient of Scheme A is below 1. As long as the economic cost is tolerable, the functional level must be improved as much as possible. For Scheme A, the most important link of choosing technical solutions is to improve the technology of the energy-saving insulation system, and increase the energysaving rate. Due to the pursuit of an energy-saving rate as high 
as $65 \%$, Scheme D has a high cost coefficient, and relatively low scores of some economic indices. In addition, this scheme merely increases the thickness of the insulation material of the external envelope structure. This practice lowers its durability, safety, and ecology performance. Hence, the value coefficient of Scheme D is smaller than 1, despite its significant advantage in applicability. To increase its value coefficient, the first step is to adjust the scheme by reducing the shape coefficient of the residential building. The walls could be supported with double layer insulation: composite wall insulation plus self-insulation. Since the external windows have reached the limit of insulation performance, some movable external shading devices could be added to enhance the comprehensive benefits. Furthermore, the parameters for the energy-saving calculation of the scheme were selected according to the provisions of Design standard for energy efficiency of residential buildings in hot summer and cold winter zone: the rated energy efficiency ratio of air-conditioning $=2.3$; the energy efficiency ratio of heating $=1.9$. If the former parameter is properly increased, the heat transfer coefficient will be lowered for the same energy-saving insulation system, according to the analysis on the relationship between heat transfer coefficient and energy consumption of the envelope structure. Then, the energy-saving rate will be properly increased, and the cost will be reduced. This is a feasible way to increase the value coefficient

\section{CONCLUSIONS}

The energy-saving techniques for the external envelope structure of residential buildings form a highly diverse system. In practice, the appropriate technology applications manifest as the composite utilization of existing techniques, including the debugging, combined use, and innovation of these techniques. The goal is to improve the overall benefits. This paper tries to make a comprehensive evaluation of the energysaving insulation system for the envelope structure of residential buildings. Firstly, the components and layers of the envelope structure were evaluated. On this basis, the value engineering principle was adopted to predict, analyze, and assess the technology, economy, and ecology of energy-saving schemes of residential buildings, trying to optimize the energy-saving performance of such buildings. The technical schemes were rated by comprehensive benefits, providing the basis for scheme selection. In addition, the comprehensive evaluation sheds light on technical improvement and reform. For some technical schemes, the overall benefits are dragged down by the imbalance between benefits of different criteria According to the weighting principle of indices, the aspects with a high weight and a low score should be improved and reformed, such as to enhance the overall technical benefits.

\section{ACKNOWLEDGEMENT}

This work is funded by the Key Project of Natural Science Research of Universities in Anhui Province (Grant No.: K2019A0821).

\section{REFERENCE}

[1] International Energy Outlook 2019, in U.S. Energy
Information Administration, 2019

[2] An, J., Yan, D., Hong, T. (2018). Clustering and statistical analyses of air-conditioning intensity and use patterns in residential buildings. Energy and Buildings, 174: 214-227.

https://doi.org/10.1016/j.enbuild.2018.06.035

[3] Pardo-Bosch, F., Cervera, C., Ysa, T. (2019). Key aspects of building retrofitting: Strategizing sustainable cities. Journal of Environmental Management, 248: 109247. https://doi.org/10.1016/j.jenvman.2019.07.018

[4] Li, G., Kou, C., Wang, H. (2019). Estimating city-level energy consumption of residential buildings: A life-cycle dynamic simulation model. Journal of Environmental Management, 240: 451-462. https://doi.org/10.1016/j.jenvman.2019.03.130

[5] Ali, U., Shamsi, M.H., Bohacek, M., Hoare, C., Purcell, K., Mangina, E., O’Donnell, J. (2020). A data-driven approach to optimize urban scale energy retrofit decisions for residential buildings. Applied Energy, 267: 114861. https://doi.org/10.1016/j.apenergy.2020.114861

[6] Azizi, S., Nair, G., Olofsson, T. (2019). Analysing the house-owners' perceptions on benefits and barriers of energy renovation in Swedish single-family houses. Energy and Buildings, 198: 187-196. https://doi.org/10.1016/j.enbuild.2019.05.034

[7] Sesana, M.M., Salvalai, G. (2018). A review on building renovation passport: potentialities and barriers on current initiatives. Energy and Buildings, 173: 195-205. https://doi.org/10.1016/j.enbuild.2018.05.027

[8] Baek, C., Park, S. (2012). Policy measures to overcome barriers to energy renovation of existing buildings. Renewable and Sustainable Energy Reviews, 16(6): 3939-3947. https://doi.org/10.1016/j.rser.2012.03.046

[9] Moschetti, R., Brattebø, H. (2016). Sustainable business models for deep energy retrofitting of buildings: state-ofthe-art and methodological approach. Energy Procedia, 96 435-445. https://doi.org/10.1016/j.egypro.2016.09.174

[10] Lee, J., Kim, J., Song, D., Kim, J., Jang, C. (2017). Impact of external insulation and internal thermal density upon energy consumption of buildings in a temperate climate with four distinct seasons. Renewable and Sustainable Energy Reviews, 75: 1081-1088. https://doi.org/10.1016/j.rser.2016.11.087

[11] Jumabekova, A., Berger, J., Foucquier, A. (2021). An efficient sensitivity analysis for energy performance of building envelope: A continuous derivative based approach. In Building Simulation, 14(4): 909-930. https://doi.org/10.1007/s12273-020-0712-4

[12] Hong, X., Shi, F., Wang, S., Yang, X., Yang, Y. (2021). Multi-objective optimization of thermochromic glazing based on daylight and energy performance evaluation. In Building Simulation, 14(6): 1685-1695. https://doi.org/10.1007/s12273-021-0778-7

[13] Na, R., Shen, Z. (2021). Assessing cooling energy reduction potentials by retrofitting traditional cavity walls into passively ventilated cavity walls. In Building Simulation, 14(4): 1295-1309. https://doi.org/10.1007/s12273-020-0740-0

[14] Ren, Z., Motlagh, O., Chen, D. (2020). A correlationbased model for building ground-coupled heat loss calculation using Artificial Neural Network techniques. Journal of Building Performance Simulation, 13(1): 4858. https://doi.org/10.1080/19401493.2019.1690581 
[15] Susorova, I., Angulo, M., Bahrami, P., Stephens, B. (2013). A model of vegetated exterior facades for evaluation of wall thermal performance. Building and Environment, 67: $1-13$. https://doi.org/10.1016/j.buildenv.2013.04.027

[16] Zhang, M., Medina, M.A., King, J.B. (2005). Development of a thermally enhanced frame wall with phase-change materials for on-peak air conditioning demand reduction and energy savings in residential buildings. International Journal of Energy Research, 29(9): 795-809. https://doi.org/10.1002/er.1082

[17] Kuusk, K., Kalamees, T., Maivel, M. (2014). Cost effectiveness of energy performance improvements in Estonian brick apartment buildings. Energy and Buildings, $\quad 77$ : 313-322 https://doi.org/10.1016/j.enbuild.2014.03.026

[18] Yu, J. (2007). Effects of envelope energy saving strategies on energy consumption in residential building. In Proceedings of the Fifth International Symposium on Heating Ventilating and Air Conditioning, pp. 849-857.

[19] Wang, Y., Huang, Z., Heng, L. (2007). Cost-effectiveness assessment of insulated exterior walls of residential buildings in cold climate. International Journal of Project Management, 25(2): 143-149. https://doi.org/10.1016/j.ijproman.2006.09.007

[20] Ghrab-Morcos, N. (2005). CHEOPS: A simplified tool for thermal assessment of Mediterranean residential buildings in hot and cold seasons. Energy and Buildings, 37(6): 651-662. https://doi.org/10.1016/j.enbuild.2004.09.020

[21] Arslanoglu, N., Yigit, A. (2017). Investigation of efficient parameters on optimum insulation thickness based on theoretical-Taguchi combined method. Environmental Progress \& Sustainable Energy, 36(6): 1824-1831. https://doi.org/10.1002/ep.12628

[22] Jebasingh, B.E., Arasu, A.V. (2020). A detailed review on heat transfer rate, supercooling, thermal stability and reliability of nanoparticle dispersed organic phase change material for low-temperature applications. Materials Today Energy, 16: 100408 https://doi.org/10.1016/j.mtener.2020.100408

[23] Gayathri, P.T.G., Shaiju, S.S., Remya, R., Deb, B. (2018). Hydrated tungsten oxide nanosheet electrodes for broadband electrochromism and energy storage. Materials Today Energy, 10: 380-387. https://doi.org/10.1016/j.mtener.2018.09.006

[24] Zakirullin, R.S. (2020). Chromogenic materials in smart windows for angular-selective filtering of solar radiation. Materials Today Energy, 17: 100476 https://doi.org/10.1016/j.mtener.2020.100476

[25] Vazquez, E., Randall, C., Duarte, J.P. (2019). Shapechanging architectural skins: A review on materials, design and fabrication strategies and performance analysis. Journal of Facade Design and Engineering, 7(2): 93-114. https://doi.org/10.7480/jfde.2019.2.3877

[26] Johari, F., Munkhammar, J., Shadram, F., Widén, J. (2022). Evaluation of simplified building energy models for urban-scale energy analysis of buildings. Building and Environment, 108684 https://doi.org/10.1016/j.buildenv.2021.108684

[27] Al-Qahtani, L.A.H., Elgizawi, L.S.E. (2020). Building envelope and energy saving case study: A residential building in Al-Riyadh, Saudi Arabia. International Journal of Low-Carbon Technologies, 15(4): 555-564. https://doi.org/10.1093/ijlct/ctaa024

[28] Ashouri, M., Astaraei, F.R., Ghasempour, R., Ahmadi, M.H., Feidt, M. (2016). Optimum insulation thickness determination of a building wall using exergetic life cycle assessment. Applied Thermal Engineering, 106: 307-315. https://doi.org/10.1016/j.applthermaleng.2016.05.190

[29] Azari, R., Garshasbi, S., Amini, P., Rashed-Ali, H., Mohammadi, Y. (2016). Multi-objective optimization of building envelope design for life cycle environmental performance. Energy and Buildings, 126: 524-534. https://doi.org/10.1016/j.enbuild.2016.05.054

[30] Biswas, K., Shrestha, S.S., Bhandari, M.S., Desjarlais, A.O. (2016). Insulation materials for commercial buildings in North America: An assessment of lifetime energy and environmental impacts. Energy and Buildings, 112: 256-269. https://doi.org/10.1016/j.enbuild.2015.12.013

[31] Dylewski, R., Adamczyk, J. (2016). Study on ecological cost-effectiveness for the thermal insulation of building external vertical walls in Poland. Journal of Cleaner Production, 133: 467-478. https://doi.org/10.1016/j.jclepro.2016.05.155

[32] Schiavoni, S., Bianchi, F., Asdrubali, F. (2016). Insulation materials for the building sector: A review and comparative analysis. Renewable and Sustainable Energy Reviews, 62: 988-1011. https://doi.org/10.1016/j.rser.2016.05.045

[33] Amiri, A., Ottelin, J., Sorvari, J. (2019). Are LEEDcertified buildings energy-efficient in practice? Sustainability, 11(6): 1672. https://doi.org/10.3390/su11061672

[34] Robati, M., Oldfield, P., Nezhad, A.A., Carmichael, D. G., Kuru, A. (2021). Carbon value engineering: A framework for integrating embodied carbon and cost reduction strategies in building design. Building and Environment, 192: 107620 . https://doi.org/10.1016/j.buildenv.2021.107620

[35] Yu, M., Robati, M., Oldfield, P., Wiedmann, T., Crawford, R., Nezhad, A.A., Carmichael, D. (2020). The impact of value engineering on embodied greenhouse gas emissions in the built environment: A hybrid life cycle assessment. Building and Environment, 168: 106452. https://doi.org/10.1016/j.buildenv.2019.106452

[36] Vijayan, R., Geetha, T.T., Nishanth, B., Tamilarasan, M., Kumar, V.V. (2019). Value engineering and value analysis of rear air spring bracket. Materials Today: Proceedings, 16: 1075-1082. https://doi.org/10.1016/j.matpr.2019.05.198

[37] Rachwan, R., Abotaleb, I., Elgazouli, M. (2016). The influence of value engineering and sustainability considerations on the project value. Procedia Environmental Sciences, 34: 431-438. https://doi.org/10.1016/j.proenv.2016.04.038

[38] Hosseini, S.M., Mohammadi, M., Rosemann, A., Schröder, T., Lichtenberg, J. (2019). A morphological approach for kinetic façade design process to improve visual and thermal comfort. Building and Environment, 153:

$186-204$. https://doi.org/10.1016/j.buildenv.2019.02.040

[39] Tabadkani, A., Roetzel, A., Li, H.X., Tsangrassoulis, A. (2021). Design approaches and typologies of adaptive facades: A review. Automation in Construction, 121: 103450. https://doi.org/10.1016/j.autcon.2020.103450 
[40] Kuru, A., Oldfield, P., Bonser, S., Fiorito, F. (2019). Biomimetic adaptive building skins: Energy and environmental regulation in buildings. Energy and Buildings, 205: https://doi.org/10.1016/j.enbuild.2019.109544

[41] Tabadkani, A., Banihashemi, S., Hosseini, M.R. (2018). Daylighting and visual comfort of oriental sun responsive skins: A parametric analysis. In Building Simulation, 11(4): 663-676. https://doi.org/10.1007/s12273-018-0433-0

[42] Todd, D., Simpson, J.A. (1983). The appropriatetechnology question in a regional context. Growth and Change, 14(4): 46-52. https://doi.org/10.1111/j.14682257.1983.tb00421.x 